\title{
Storage related haematological and biochemical changes in Plasmodium falciparum infected and sickle cell trait donor blood
}

\author{
Enoch Aninagyei ${ }^{*^{*}} \mathbb{D}$, Emmanuel Tetteh Doku², Patrick Adu ${ }^{3}$, Alexander Egyir-Yawson ${ }^{1}$
} and Desmond Omane Acheampong ${ }^{1 *}$

\begin{abstract}
Background: In sub-Saharan Africa where sickle cell trait (SCT) and malaria is prevalent, significant proportions of blood donors may be affected by one or more of these abnormalities. The haemato-biochemical properties of SCT and asymptomatic malaria in donor blood have not been evaluated. This study evaluated the haemato-biochemical impact of SCT and asymptomatic malaria infections in citrate-phosphate-dextrose-adenine (CPDA-1) stored donor blood units.

Methods: Fifty-milliliters of sterile CPDA-1 anti-coagulated blood were drained into the sample pouch attached to the main blood bag. Ten units each of sickle cell/malaria negative, sickle cell and malaria positive blood were analyzed. Baseline and weekly haematological profiling and week 1, 3 and 5 concentrations of plasma haemoglobin, \% haemolysis, sodium, potassium and chloride and lactate dehydrogenase $(\mathrm{LDH})$ were assayed. Differences between baseline and weekly data were determined using one-way analysis of variance (ANOVA) and Kruskal-Wallis test, whereas differences between baseline parameters and week 1-3 data pairs were determined using paired t-test. $P$ value $<0.05$ was considered statistically significant.
\end{abstract}

Results: Storage of SCT and malaria infected blood affected all haematological cell lines. In the SCT donors, red blood cells (RBC) $\left(4.75 \times 10^{12} / \mathrm{L} \pm 1.43^{\text {baseline }}\right.$ to $\left.3.49 \times 10^{12} / \mathrm{L} \pm 1.09^{\text {week-5}}\right)$, haemoglobin $\left(14.45 \mathrm{~g} / \mathrm{dl} \pm 1.63^{\text {baseline }}\right.$ to $11.43 \mathrm{~g} / \mathrm{dl}$ $\left.\pm 1.69^{\text {week-5}}\right)$ and haematocrit $\left(39.96 \% \pm 3.18^{\text {baseline }}\right.$ to $\left.33.22 \% \pm 4.12^{\text {week-5}}\right)$ were reduced. In the asymptomatic malaria group, reductions were observed in RBC $\left(5.00 \times 10^{12} / \mathrm{L} \pm 0.75^{\text {baseline }}\right.$ to $\left.3.72 \times 10^{12} / \mathrm{L} \pm 0.71^{\text {week-5 }}\right)$, haemoglobin $\left(14.73 \mathrm{~g} / \mathrm{dl} \pm 1.67^{\text {baseline }}\right.$ to $\left.11.53 \mathrm{~g} / \mathrm{dl} \pm 1.62^{\text {week-5}}\right)$, haematocrit $\left(42.72 \% \pm 5.16^{\text {baseline }}\right.$ to $\left.33.38 \% \pm 5.80^{\text {week-5}}\right)$, mean cell haemoglobin concentration $\left(35.48 \mathrm{~g} / \mathrm{dl} \pm 1.84^{\text {baseline }}\right.$ to $\left.35.01 \mathrm{~g} / \mathrm{dl} \pm 0.64^{\text {week-5}}\right)$ and red cell distribution width coefficient of variation $\left(14.81 \% \pm 1.54^{\text {baseline }}\right.$ to $16.26 \% \pm 1.37^{\text {week-5 }}$ ). Biochemically, whereas plasma LDH levels significantly increased in asymptomatic malaria blood donors (319\% increase at week 5 compared to baseline), SCT blood donors had the most significant increase in plasma potassium levels at week 5 (382\% increase). Sodium ions significantly reduced in SCT/malaria negative and sickle cell trait blood at an average rate of $0.21 \mathrm{mmol} / \mathrm{L}$ per day. Moreover, elevations in lymphocytes-to-eosinophils and lymphocytes-to-neutrophils ratios were associated with SCT and malaria positive blood whilst elevation lymphocytes-to-basophils ratio was exclusive to malaria positive blood.

Conclusion: Severe storage lesions were significant in SCT or malaria positive donor blood units. Proper clinical evaluation must be done in prospective blood donors to ensure deferral of such donors.

Keywords: Sickle cell trait donor, Asymptomatic malaria donor, \% haemolysis, Storage lesions, Plasma haemoglobin, Biochemical changes, Blood transfusion

\footnotetext{
* Correspondence: enochaninagyei@yahoo.com; dacheampong@ucc.edu.gh

'Department of Biomedical Sciences, School of Allied Health Sciences,

University of Cape Coast, Cape Coast, Ghana

Full list of author information is available at the end of the article
}

(c) The Author(s). 2018 Open Access This article is distributed under the terms of the Creative Commons Attribution 4.0 International License (http://creativecommons.org/licenses/by/4.0/), which permits unrestricted use, distribution, and reproduction in any medium, provided you give appropriate credit to the original author(s) and the source, provide a link to the Creative Commons license, and indicate if changes were made. The Creative Commons Public Domain Dedication waiver (http://creativecommons.org/publicdomain/zero/1.0/) applies to the data made available in this article, unless otherwise stated. 


\section{Background}

There are two major outcomes of Plasmodium infections, thus symptomatic and asymptomatic infections. Symptomatic infections occur in patients with compromised anti-disease immunity [1] and asymptomatic infections occur in individuals with competent malaria immunity [2]. Despite being asymptomatic, parasites may be present in red blood cells, but at low density and can persist for many months [3]. Prevalence of asymptomatic Plasmodium infections differs from one geographical region to another. In Brazil, Solomon Islands and Cambodia, asymptomatic malaria has been found to be $37.5,82.2$ and $92 \%$ respectively [4-6]. In Africa, the prevalence of asymptomatic malaria in blood donors in Ghana has been found to be $10.0 \%$ [7]. In Cameroon, Senegal, Benin and Nigeria, asymptomatic malaria parasitaemia in blood donors have been found to be $27.54 \%$ [8], 65.3\% [9], > 30\% [10] and 40\% [11] respectively.

Sickle cell haemoglobin result when valine substitutes for glutamic acid at position 6 of the $\beta$-globin chain. This mutation consequently changes the physico-chemical properties of the haemoglobin molecule [12]. A person who inherits haemoglobin S from one parent and haemoglobin A (normal gene) from the other has a sickle cell trait (SCT) or is said to be a carrier of sickle cell disease [13]. The prevalence of sickle cell trait (SCT) is highest in Africans and people of African descent. In Africa, Nigeria probably has the highest number of people with SCT as close to $30 \%$ of the population carry haemoglobin S gene [14]. Liberia, Ghana and Uganda have $10-15 \%$ of their population being carriers of haemoglobin $S$ gene $[13,15,16]$. Cameroon and Gabon have haemoglobin S gene prevalence of 19 and $22 \%$, respectively [17, 18]. SCT individuals are clinically healthy to donate blood [19]. Populations with high prevalence of sickle cell trait and asymptomatic Plasmodium falciparum infections can result in high number of healthy blood donors with sickle cell trait and/or asymptomatic Plasmodium infections. In Kenya and Nigeria, proportion of their blood donor population who were sickle cell carriers were 3.9 and $27.1 \%$ respectively [20, 21]. In Ghana, studies done in Brong Ahafo and Greater Accra regions reported prevalence of $\mathrm{SCT}$ in blood donors to be 12.5 and $11.3 \%$ respectively [12, 22].

Majority of the blood banks in SSA still practice whole blood banking [23, 24]. Although donated blood units are stored in anticoagulants supplemented with phosphate, dextrose, and/or adenine to assure long-term viability, storage lesions are inevitable when blood is stored for long periods [25]. RBC storage lesions include decreased RBC stability, alterations in various metabolites and the metabolic status of the cell, including decrease of intracellular adenosine triphosphate (ATP) and 2,3-biphosphoglycerate (2,3 BPG) [26-28]. These changes in RBC during storage have been known for years but the exact changes conferred on blood collected from SCT donors and those infected with Plasmodium parasites are unknown. The aim of this study therefore was to evaluate, for the first time, the haematological and biochemical variations in sickle cell trait blood and blood infected with Plasmodium falciparum stored in CPDA-1 anticoagulant up to 35 days.

\section{Methods}

Study design

This cross-sectional laboratory based experimental study was done in sterile blood units. Donors were selected according to Medical History Guide for Donor Selection [29].

\section{Donor selection, phlebotomy and specimen collection}

The selections of the healthy blood donors were double-blinded as researchers and National Blood Service Ghana staff could not link the study specimens to any donor. Participant consent form was signed by all participated donors. Blood was collected from each of the donors following a modification of the technique described by Cheesbrough [25]. For the purpose of the study, $7 \mathrm{ml}$ of the CDPA-1 anticoagulant was allowed into the sample pouch attached to the main blood bag, which was filled with the initial $50 \mathrm{ml}$ of whole blood. The rest of the whole blood was directed into the main blood bag.

\section{Inclusion criteria}

Donor blood included in the study were TTIs-negative blood with baseline biochemical parameters that fell within these ranges; haemoglobin $12.5-18.00 \mathrm{~g} / \mathrm{dl}$, plasma haemoglobin $-0 \mathrm{~g} / \mathrm{dl}$, percentage haemolysis- $0.0 \%$, plasma sodium, potassium, chloride and LDH that fell within these respective ranges $135-145 \mathrm{mmo} / \mathrm{l}, 3.0-5.2 \mathrm{mmol} / \mathrm{l}$, $95-108 \mathrm{mmol} / \mathrm{l}$ and $100-250 \mathrm{U} / \mathrm{L}$.

\section{Laboratory procedures}

Post-phlebotomy laboratory procedures were done as follows: $5 \mathrm{ml}$ of well mixed whole blood was aspirated into plain glass vacutainer tubes (All-Pro, Dusseldorf, Germany). Portion of the blood was used to screen for transfusion-transmitted infections (TTIs) using fourth generation enzyme immuno-assay (Abnova, Taiwan). The ELISA microtitre plate wells were pre-coated with hepatitis B surface monoclonal antibodies, gp36/gp41, hepatitis C antigens (core, NS3 and NS5) and Treponema pallidum antigens for qualitative detection of hepatitis B virus, HIV I\&II, hepatitis C virus and Treponema pallidum respectively. Donor screening of sickle cells was done as described by Antwi-Baffour [13] whilst phenotyping was done in alkaline medium $(\mathrm{pH}=8.6)$. Separation of haemolysate was done on cellulose acetate paper using $250 \mathrm{~V}$ voltage and $50 \mathrm{~mA}$ current for 
$30 \mathrm{~min}$. The separated haemoglobin molecules were interpreted against pooled haemoglobin AFSC controls. Screening of malaria was done with PfHRP-2/Pf-LDH SD Bioline rapid diagnostic test kit (Gyeonggi-do, Republic of Korea). The kit detected Plasmodium falciparum specific HRP-2 proteins and lactate dehydrogenase enzymes. Five microliters of whole blood were dropped on the sample column of the rapid test kit. Four drops of assay diluent (PfHRP-2/Pf-LDH SD Bioline, Korea) were added to the buffer window of the kit. Results were read after $15 \mathrm{~min}$. Baseline haematological parameters using 5-part differential Urit-5160 (Guangxi, China), plasma electrolytes (sodium, potassium and chloride) measured by FT-320 electrolyte analyzer (China), lactate dehydrogenase (measured by PKL-125 Italia fully automated chemistry analyzer using ELItech LDH kinetic reagent, France), plasma haemoglobin (Urit-5160, China) and percentage haemolysis calculated by using formulae used by Sawant et al. [30] was done on same day of blood collection (baseline). The donor whole blood study samples were stored in the blood bank refrigerator (Fiocchetti Scientific Refrigerator, Italy) for 5 weeks. Internal storage temperature was taken twice daily. Haematological parameters were determined weekly for 5 weeks whilst plasma haemoglobin, \% haemolysis, plasma electrolytes and LDH were measured at the end of week 1 , week 3 and week 5 .

\section{Data processing and statistical analysis}

Baseline and weekly data was entered into Microsoft Excel 2010. Statistical analysis was done by SPSS Version 24 (Chicago, IL, USA). Based on SCT status and/or asymptomatic malaria infection status, participants were grouped into three: SCT blood donors, asymptomatic malaria blood donors and no SCT/asymptomatic malaria blood donors for subsequent analysis. Differences between baseline and the 5 weeks haematological profile and biochemical data set were determined by one-way analysis of variance (ANOVA) and Kruskal-Wallis test statistical models. Differences between baseline haematological parameters and week 1 to 3 data pair were determined by paired student T-test. $P$-value of $<0.05$ was considered statistically significant.

\section{Results}

\section{Storage related changes in TWBC and WBC differential counts}

The study recorded significant changes in total white blood cells (TWBC) and WBC differential in all the three donor groups (Table 1). There were consecutive reductions in TWBC and neutrophil proportion in all donor groups. Whereas the largest significant TWBC reduction occurred in the sickle cell/malaria negative group (62.3\% reduction in week 5), the largest significant reduction in neutrophil\% occurred in the SCT donors (53.9\% reduction in week 5). However, there were successive increases in \%lymphocytes, basophils and monocytes in all donor groups when weekly estimates were compared to baseline values. Whereas the highest increase in \%lymphocytes occurred in the sickle cell/malaria negative donors (84.3\% increase compared to baseline), the highest \%monocyte increase occurred in the SCT donor group ( $75.6 \%$ increase compared to baseline). Additionally, although there was a consecutive reduction in \%eosinophil count in the SCT group, the changes in \%eosinophil count fluctuated in the sickle cell/malaria negative, and the asymptomatic malaria donor groups.

\section{Storage related changes in $\mathrm{RBC}$ and $\mathrm{RBC}$ indices}

This study also found significant changes in RBC count and RBC indices in all the three donor groups (Table 2). In all donor groups, there were consecutive reductions of RBC count, haemoglobin level, and HCT in all successive weekly estimations compared to baseline values. Whereas the most significant reduction in $\mathrm{RBC}$ count occurred in the SCT blood donor group, (26.5\% decrease compared to baseline), the highest reductions in haemoglobin and HCT occurred in the sickle cell/malaria negative blood donor group [26.5\% $(\mathrm{Hb})$, and $25.2 \%$ (HCT) reductions compared to baseline]. Also, RDW-CV\% values consecutively increased in all donor groups with respect to successive weekly measurement. However, whereas weekly $\mathrm{MCV}$ and $\mathrm{MCH}$ values consistently decreased in asymptomatic malaria donor group, these values fluctuated in SCT or sickle cell/malaria negative donor groups.

\section{Storage related changes in platelets and platelet indices}

Changes in platelet count and related platelet indices were also observed in the donor population (Table 3). Consecutively, weekly platelet count estimates successively decreased in all blood donor groups; the highest significant reduction occurring in asymptomatic malaria blood donor group (49.9\% decrease compared to baseline). Also, whereas weekly MPV and P_LCR successively increased in sickle cell/malaria negative and asymptomatic malaria donor group, the levels of these measurements fluctuated in the SCT blood donor group.

\section{Storage related changes in leukocyte ratios}

There was progressive increase in lymphocyte -to-eosinophils ratio (LER) in the three blood donor groups but the differences in the SCT/malaria negative group were not significant $(\% \Delta 4.0-20.7 \%, \mathrm{~F}=$ 2.363, $p=0.052$ ) whilst significant differences were seen in the SCT $(\% \Delta 55.9-178 \%, \mathrm{~F}=5.16, p=<0.05)$ and the malaria positive donor group (\% $\%$ 35.5$514.7 \%, \quad \mathrm{~F}=4.46, p=<0.05)$. In addition to LER, 
Table 1 Leukocytes and percentage differential storage changes in donor blood

\begin{tabular}{|c|c|c|c|c|c|c|c|}
\hline $\begin{array}{l}\text { TWBC and } \\
\text { differentials }\end{array}$ & $\begin{array}{l}\text { Baseline } \\
\text { Mean }\end{array}$ & $\begin{array}{l}\text { Week } 1 \\
\text { Mean }(\% \Delta)\end{array}$ & $\begin{array}{l}\text { Week } 2 \\
\text { Mean }(\% \Delta)\end{array}$ & $\begin{array}{l}\text { Week } 3 \\
\text { Mean }(\% \Delta)\end{array}$ & $\begin{array}{l}\text { Week } 4 \\
\text { Mean }(\% \Delta)\end{array}$ & $\begin{array}{l}\text { Week } 5 \\
\text { Mean }(\% \Delta)\end{array}$ & $P$-value \\
\hline \multicolumn{8}{|c|}{ Sickle cell/malaria negative donor blood } \\
\hline $\operatorname{TWBC} \times 10^{9} / \mathrm{L}$ & 5.63 & $3.89(-30.9)$ & $2.90(-48.4)$ & $2.49(-55.7)$ & $2.27(-59.6)$ & $2.09(-62.3)$ & $0.001^{*}, \mathrm{a}$ \\
\hline Neut \% & 66.18 & $60.09(-9.2)$ & $52.77(-20.2)$ & $49.49(-25.2)$ & $44.21(-33.2)$ & $40.88(-38.2)$ & $0.054^{b}$ \\
\hline Lymp \% & 27.28 & $33.42(22.5)$ & $39.83(46.0)$ & $41.92(53.6)$ & 46.94(72.0) & $50.28(84.3)$ & $0.092^{b}$ \\
\hline Eos $\%$ & 3.03 & $2.73(-9.9)$ & $2.42(-20.1)$ & $3.32(9.5)$ & $3.00(-0.9)$ & $3.49(15.2)$ & $0.845^{\mathrm{a}}$ \\
\hline Mon \% & 3.27 & $3.52(7.6)$ & $4.64(41.9)$ & $4.94(51.1)$ & $5.50(68.2)$ & $4.96(51.6)$ & $0.009^{b}$ \\
\hline Bas \% & 0.24 & $0.25(4.1)$ & $0.35(45.8)$ & $0.33(37.5)$ & $0.35(45.8)$ & $0.39(62.5)$ & $0.033^{b}$ \\
\hline \multicolumn{8}{|c|}{ Sickle cell trait donor blood } \\
\hline $\operatorname{TWBC} \times 10^{9} / \mathrm{L}$ & 6.36 & $5.08(-20.1)$ & $4.42(-30.5)$ & $4.24(-33.3)$ & $3.74(-41.2)$ & $3.57(-43.8)$ & $0.001^{*}$,b \\
\hline Neut $\%$ & 56.26 & $50.91(-9.5)$ & 39.58(-29.6) & $32.64(-41.9)$ & $31.99(-43.1)$ & $25.90(-53.9)$ & $0.001^{*}, \mathrm{~b}$ \\
\hline Lymp \% & 37.50 & $43.06(14.8)$ & $51.48(37.3)$ & $61.46(63.9)$ & $62.79(67.4)$ & $66.28(76.7)$ & $0.001^{*}, 1$ \\
\hline Eos \% & 3.34 & $2.45(-26.6)$ & $2.50(-25.1)$ & $1.56(-53.3)$ & $1.69(-49.4)$ & $2.77(-17.0)$ & $0.020^{\mathrm{a}}$ \\
\hline Mon \% & 2.71 & $3.59(32.4)$ & $3.94(45.4)$ & $4.02(48.3)$ & $3.24(19.5)$ & $4.76(75.6)$ & $0.179^{\mathrm{b}}$ \\
\hline Bas \% & 0.20 & $0.20(0.0)$ & $0.38(90.0)$ & $0.32(60.0)$ & $0.30(50.0)$ & $0.29(45.0)$ & $0.069^{b}$ \\
\hline \multicolumn{8}{|c|}{ Asymptomatic malaria donor blood } \\
\hline$T W B C \times 10^{9} / L$ & 6.08 & $4.49(-26.1)$ & $4.13(-32.1)$ & $3.77(-37.9)$ & $3.54(-41.7)$ & $3.21(-47.2)$ & $0.001^{\mathrm{a}}$ \\
\hline Neut $\%$ & 72.09 & $60.89(-15.5)$ & $47.78(-33.7)$ & $39.79(-44.8)$ & $42.91(-40.4)$ & $36.07(-49.9)$ & $0.001^{*}, \mathrm{~b}$ \\
\hline Lymp \% & 31.67 & $32.53(2.7)$ & $45.65(44.1)$ & $52.53(65.8)$ & $49.52(56.3)$ & $55.63(75.6)$ & $0.001^{*}, \mathrm{~b}$ \\
\hline Eos $\%$ & 2.38 & $2.50(5.0)$ & $2.01(-15.5)$ & $2.26(-5.0)$ & $2.39(0.4)$ & $2.69(13.0)$ & $0.265^{a}$ \\
\hline Mon \% & 3.56 & $3.72(4.5)$ & $4.68(31.4)$ & $5.08(42.6)$ & $4.57(28.4)$ & $5.29(48.6)$ & $0.095^{b}$ \\
\hline Bas \% & 0.30 & $0.36(20.0)$ & $0.33(10.0)$ & $0.34(13.3)$ & 0.31 (3.3) & $0.32(6.6)$ & $0.804^{a}$ \\
\hline
\end{tabular}

Abbreviations: $T W B C=$ total white blood cells, Neut $=$ Neutrophils, $L y m p=$ Lymphocytes, Eos = Eosinophils, Mon = Monocytes, Bas $=$ Basophils, $\%=$ Percent $L=$ Liter, $A N O V A=$ Analysis of variance, $S D=$ Standard deviation

${ }^{*} p$ values less than $0.001,{ }^{a} p$-value determined by Kruskal-Wallis $\mathrm{H}$ test, ${ }^{b} p$-value determined by one-way ANOVA

significant increase in lymphocyte-to-basophils ratio (LBR) was observed in malaria positive donor group $(\% \Delta 25.3-151.9 \%, \mathrm{~F}=5.11, p=<0.05)$ but not in SCT group $(\mathrm{F}=0.73, p=0.602)$ and $\mathrm{SCT} /$ malaria negative group $(\mathrm{F}=0.34, p=0.882)$. There were gradual increases in lymphocytes-to-monocytes ratio (LMR) in both the SCT and the malaria positive group but the differences between the values were not significant. Lymphocyte-to-neutrophil ratio (LNR) increased in the three groups but significant increases were seen in SCT $(29.1-308.8 \%, \mathrm{~F}=4.53, p=0.001)$ and malaria positive blood (75-437.5\%, F = 10.9, $p<0.05$ ) (Table 4).

Moreover, when the weekly estimates in haematological parameters were compared to baseline using paired $t$-test analyses, most of the significant changes occurred in the SCT blood donors or the asymptomatic malaria positive blood donors (Additional file 1: Table S1).

\section{Effect of storage on haemolytic and biochemical parameters in donor blood}

The storage lesions related to haemolytic and biochemical parameters presented in Table 5. The plasma haemoglobin and \%haemolysis consecutively increased with the successive weekly estimations in all blood donor groups. Additionally, weekly LDH and plasma potassium levels consecutively increased in weekly estimates compared to baseline in all blood donor groups. Whereas the most significant increase in LDH occurred in asymptomatic malaria blood donor group (319\% increase over baseline), the most significant increase in plasma potassium levels occurred in SCT blood donor group (382\% increase over baseline levels). However, weekly plasma sodium and chloride levels successively decreased in all blood donor group; the highest significant reductions occurring in asymptomatic malaria blood donor group [10.0\% (sodium) and $21.3 \%$ (chloride) decreases compared to baseline].

\section{Comparison of haemolytic and biochemical parameters across blood donor groups}

The storage lesions in donor blood were quantitatively compared across the groups with respect to the weeks in storage (Table 6). Plasma haemoglobin and \%haemolysis were significantly higher in SCT blood donors with respective weekly measurements compared to the other blood donor groups. However, respective weekly plasma 
Table 2 RBC and RBC indices storage changes in donor blood

\begin{tabular}{|c|c|c|c|c|c|c|c|}
\hline $\begin{array}{l}\text { RBC and } \\
\text { RBC indices }\end{array}$ & $\begin{array}{l}\text { Baseline } \\
\text { Mean }\end{array}$ & $\begin{array}{l}\text { Week } 1 \\
\text { Mean }(\% \Delta)\end{array}$ & $\begin{array}{l}\text { Week } 2 \\
\text { Mean }(\% \Delta)\end{array}$ & $\begin{array}{l}\text { Week } 3 \\
\text { Mean }(\% \Delta)\end{array}$ & $\begin{array}{l}\text { Week } 4 \\
\text { Mean }(\% \Delta)\end{array}$ & $\begin{array}{l}\text { Week } 5 \\
\text { Mean }(\% \Delta)\end{array}$ & $P$-values \\
\hline \multicolumn{8}{|c|}{ Sickle cell and malaria negative donor blood } \\
\hline $\mathrm{RBC}\left(\times 10^{12} / \mathrm{L}\right)$ & 4.72 & $4.27(-9.5)$ & $4.08(-13.5)$ & $4.03(-14.6)$ & $3.98(-15.6)$ & $4.04(-14.4)$ & $0.147^{\mathrm{a}}$ \\
\hline $\mathrm{Hb}(\mathrm{g} / \mathrm{dl})$ & 13.17 & $11.35(-13.8)$ & $10.49(-20.3)$ & $9.97(-24.3)$ & $10.06(-23.6)$ & $9.74(-26.0)$ & $0.022^{\mathrm{a}}$ \\
\hline HCT (\%) & 37.54 & $33.52(-10.7)$ & $28.83(-23.2)$ & $28.52(-24.0)$ & $28.48(-24.1)$ & $28.06(-25.2)$ & $0.013^{\mathrm{a}}$ \\
\hline MCV (fL) & 75.88 & $76.99(1.5)$ & $75.34(-0.7)$ & $74.37(-1.9)$ & $74.67(-1.6)$ & $75.22(-0.9)$ & $0.952^{\mathrm{a}}$ \\
\hline $\mathrm{MCH}(\mathrm{pg})$ & 26.70 & $26.23(-1.7)$ & $27.61(3.4)$ & $25.85(-3.1)$ & $26.39(-1.1)$ & $26.14(-2.0)$ & $0.924^{\mathrm{a}}$ \\
\hline $\mathrm{MCHC}(\mathrm{g} / \mathrm{dl})$ & 32.47 & $31.39(-3.3)$ & $33.58(3.4)$ & $31.90(-1.7)$ & $32.45(-0.6)$ & $31.92(-1.6)$ & $0.932^{\mathrm{a}}$ \\
\hline RDW_CV (\%) & 14.14 & $14.14(0.0)$ & $14.20(0.4)$ & $15.02(6.2)$ & $14.63(3.4)$ & $14.76(4.3)$ & $0.486^{\mathrm{b}}$ \\
\hline RDW_SD (fL) & 32.38 & $32.38(0.0)$ & $33.55(3.6)$ & $31.76(-1.9)$ & $32.14(-0.7)$ & $30.17(-6.8)$ & $0.756^{b}$ \\
\hline \multicolumn{8}{|c|}{ Sickle cell trait donor blood } \\
\hline $\mathrm{RBC}\left(\times 10^{12} / \mathrm{L}\right)$ & 4.75 & $4.06(-14.5)$ & $3.58(-24.6)$ & $3.57(-24.8)$ & $3.56(-25.0)$ & $3.49(-26.5)$ & $0.016^{\mathrm{a}}$ \\
\hline $\mathrm{Hb}(\mathrm{g} / \mathrm{dl})$ & 14.45 & $12.84(-11.1)$ & $12.47(-13.7)$ & $11.47(-20.6)$ & $11.57(-19.9)$ & $11.43(-20.8)$ & $0.002^{\mathrm{a}}$ \\
\hline HCT (\%) & 39.96 & $36.75(-8.0)$ & $33.34(-16.5)$ & $33.29(-16.7)$ & $32.55(-18.5)$ & $33.22(-16.8)$ & $0.005^{\mathrm{a}}$ \\
\hline MCV (fL) & 82.54 & $86.71(5.0)$ & $82.02(-0.6)$ & $81.27(-1.5)$ & $82.03(-0.61)$ & $82.95(0.4)$ & $0.573^{\mathrm{a}}$ \\
\hline $\mathrm{MCH}(\mathrm{pg})$ & 30.36 & $30.29(-0.2)$ & $30.39(0.3)$ & $28.54(-5.9)$ & $29.12(-4.0)$ & $28.43(-6.3)$ & $0.424^{\mathrm{a}}$ \\
\hline $\mathrm{MCHC}(\mathrm{g} / \mathrm{dl})$ & 36.75 & $34.99(-4.7)$ & $37.08(0.9)$ & $34.96(-4.8)$ & $35.50(-3.4)$ & $34.30(-6.6)$ & $0.001^{a}$ \\
\hline RDW_CV (\%) & 14.75 & $14.75(0.0)$ & $14.91(1.0)$ & $15.30(3.7)$ & $15.03(1.8)$ & $23.68(60.5)$ & $0.518^{b}$ \\
\hline RDW_SD (fL) & 35.59 & $35.59(0.0)$ & $36.54(2.6)$ & $33.80(5.0)$ & $34.06(4.2)$ & $31.83(10.5)$ & $0.082^{b}$ \\
\hline \multicolumn{8}{|c|}{ Asymptomatic malaria donor blood } \\
\hline $\mathrm{RBC}\left(\times 10^{12} / \mathrm{L}\right)$ & 5.00 & $4.48(-10.4)$ & $4.02(-19.6)$ & $4.00(-20.0)$ & $3.96(-20.8)$ & $3.72(-25.6)$ & $0.001^{*}, a$ \\
\hline $\mathrm{Hb}(\mathrm{g} / \mathrm{dl})$ & 14.73 & $13.06(-11.3)$ & $12.12(-17.7)$ & $11.82(-19.7)$ & $11.98(-18.6)$ & $11.53(-21.7)$ & $0.001^{*}, a$ \\
\hline HCT (\%) & 42.72 & $38.83(-9.1)$ & $34.04(-20.3)$ & $34.32(-19.6)$ & $34.33(-19.6)$ & $33.38(-21.8)$ & $0.001^{*}, a$ \\
\hline MCV (fL) & 89.15 & $87.66(-1.6)$ & $82.04(-7.9)$ & $81.81(-8.2)$ & $82.68(-7.2)$ & $83.31(-6.5)$ & $0.067^{\mathrm{a}}$ \\
\hline $\mathrm{MCH}(\mathrm{pg})$ & 30.85 & $29.42(-4.6)$ & $29.66(-3.8)$ & $28.13(-8.8)$ & $29.20(-5.3)$ & $29.15(-5.5)$ & $0.320^{\mathrm{a}}$ \\
\hline $\mathrm{MCHC}(\mathrm{g} / \mathrm{dl})$ & 35.48 & $33.57(-5.3)$ & $36.22(2.0)$ & $34.45(2.9)$ & $35.38(-0.2)$ & $35.01(-1.3)$ & $0.002^{b}$ \\
\hline RDW_CV (\%) & 14.81 & $14.81(0.0)$ & $15.80(6.6)$ & $16.39(10.6)$ & $16.16(9.1)$ & $16.26(9.7)$ & $0.024^{\mathrm{a}}$ \\
\hline RDW_SD (fL) & 36.53 & $36.53(0.0)$ & $35.75(-2.1)$ & $33.82(-7.4)$ & $34.49(-5.5)$ & $34.49(-5.5)$ & $0.267^{a}$ \\
\hline
\end{tabular}

Abbreviations: $R B C=$ Red blood cells, $H b=$ Haemoglobin, $H C T=$ Haematocrit,$M C V=$ Mean cell volume, $M C H=$ Mean cell haemoglobin, $M C H C=$ Mean cell haemoglobin concentration, $R D W_{-} C V=$ Red cell distribution width coefficient of variation, $R D W \_S D=$ Red cell distribution width standard deviation, $L=L$ itre, $f L=$ Fentolitre,$p g=$ picogram

${ }^{*} p$ values less than $0.001 ;{ }^{a} p$-value determined by one-way ANOVA; ${ }^{b} p$-value determined by Kruskal-Wallis $\mathrm{H}$ test

LDH levels were significantly higher in asymptomatic malaria blood donor group compared to respective values in the other blood donor groups. Additionally, although baseline plasma potassium levels were comparable in all blood donor groups, weekly plasma potassium levels were respectively higher in asymptomatic malaria or SCT blood donor group compared to sickle cell and malaria negative blood donor group. Moreover, plasma sodium and chloride significantly decreased in the asymptomatic malaria group compared to the other groups.

\section{Discussion}

Preservation and long term storage of red blood cells is essential for continuous supply of safe blood for clinical use [24]. Previous studies have found deleterious storage effect on blood cell morphology and functions [31-33]. This study found similar trend of storage lesion in whole blood stored up to 5 weeks but the changes were more pronounced in blood collected from sickle cell trait (SCT) donors or donors infected asymptomatically with malaria parasites. In the three groups, gradual reduction in total white cells and neutrophils and progressive increase in lymphocytes were observed; a phenomenon that was previously observed by Adias [24]. This observation probably can be due to cell loss and cytotoxic effect of histamine and cytokines released by neutrophils [34]. TWBC, neutrophils and eosinophils cells lost were more evident in the SCT or asymptomatic malaria positive stored blood than sickle cell and malaria negative blood. It was observed that lymphocytes to eosinophils, 
Table 3 Platelet and platelet indices storages changes in donor blood

\begin{tabular}{|c|c|c|c|c|c|c|c|c|}
\hline & Baseline Mean & Week 1 Mean $(\% \Delta)$ & Week 2 Mean $(\% \Delta)$ & Week 3 Mean $(\% \triangle)$ & Week 4 Mean $(\% \Delta)$ & Week 5 Mean $(\% \triangle)$ & $\mathrm{F}$ & $P$-value \\
\hline \multicolumn{9}{|c|}{ Sickle cell and malaria negative donor blood } \\
\hline Plt $\left(\times 10^{9} / L\right)$ & 224.10 & 199.55(10.9) & $171.10(23.6)$ & $162.60(27.4)$ & $167.40(25.3)$ & 139.30(37.8) & 4.09 & 0.003 \\
\hline MPV (fL) & 8.45 & $9.05(7.1)$ & $9.93(17.5)$ & $9.71(14.9)$ & $9.17(8.5)$ & $10.10(19.5)$ & 8.18 & $0.001^{*}$ \\
\hline PDW (fL) & 12.74 & $12.41(2.5)$ & 13.93(9.3) & $13.00(2.0)$ & 11.98(5.9) & $13.63(6.9)$ & 1.00 & 0.425 \\
\hline PCT (\%) & 0.18 & $0.16(11.1)$ & $0.17(5.5)$ & $0.15(16.6)$ & $0.15(16.6)$ & $0.13(27.7)$ & 1.09 & 0.374 \\
\hline P_LCR (\%) & 18.15 & $20.11(10.7)$ & $21.86(20.4)$ & $22.15(22.0)$ & $19.21(5.8)$ & $24.18(33.2)$ & 1.24 & 0.304 \\
\hline \multicolumn{9}{|c|}{ Sickle cell trait donor blood } \\
\hline Plt $\left(\times 10^{9} / \mathrm{L}\right)$ & 225.90 & $214.10(5.2)$ & $185.20(18.0)$ & $189.40(16.1)$ & 207.80(8.0) & 167.61(25.8) & 1.25 & 0.301 \\
\hline MPV (fL) & 8.69 & $8.53(1.8)$ & $9.83(13.1)$ & $9.78(12.5)$ & $9.83(13.1)$ & $10.45(20.2)$ & 3.85 & 0.005 \\
\hline PDW (fL) & 12.31 & $12.03(2.2)$ & 13.89(12.8) & 13.75(11.6) & $13.72(11.4)$ & $15.54(26.2)$ & 1.55 & 0.189 \\
\hline PCT (\%) & 0.19 & $0.19(0.0)$ & $0.18(5.2)$ & $0.18(5.2)$ & $0.20(5.2)$ & $0.18(5.2)$ & 0.31 & 0.903 \\
\hline P_LCR (\%) & 16.62 & $16.19(2.5)$ & 20.02(20.4) & $21.65(30.2)$ & $20.35(22.4)$ & $24.28(46.0)$ & 2.09 & 0.080 \\
\hline \multicolumn{9}{|c|}{ Asymptomatic malaria donor blood } \\
\hline Plt $\left(\times 10^{9} / L\right)$ & 191.90 & $141.30(26.3)$ & $132.70(30.8)$ & $118.40(38.3)$ & 106.80(44.3) & $96.10(49.9)$ & 7.47 & $0.001^{*}$ \\
\hline MPV (fL) & 8.59 & $8.59(0.0)$ & $9.87(14.9)$ & $9.93(15.6)$ & $9.10(5.9)$ & 10.19(18.6) & 2.52 & 0.040 \\
\hline PDW (fL) & 13.90 & $13.90(0.0)$ & $14.28(2.7)$ & $14.75(6.1)$ & $12.25(11.8)$ & $15.98(14.9)$ & 1.49 & 0.208 \\
\hline PCT (\%) & 0.15 & $0.15(0.0)$ & $0.16(6.6)$ & $0.15(0.0)$ & $0.16(6.6)$ & $0.14(6.6)$ & 0.41 & 0.835 \\
\hline P_LCR (\%) & 19.48 & $19.48((0.0)$ & 22.19(13.9) & $22.17(13.8)$ & 21.40(9.8) & $26.89(38.0)$ & 1.33 & 0.265 \\
\hline
\end{tabular}

Abbreviations: $P l t=$ Platelets, $P M V=$ Mean platelet volume, $P D W=$ Platelet distribution width, $P C T=$ Plateletcrit, $P \_L C R=$ Platelet large cell ratio ${ }^{*} p$ values less than 0.001

monocytes, basophils and neutrophils ratios were elevated in the three groups but in all cases significant elevations occurred in SCT and malaria positive donor blood. Insignificant elevations were observed in SCT/ malaria negative blood. Elevation in LER, LMR, LBR and
LNR values occurred as a result of progressive elevations in mean lymphocytes percentages and reduction in mean absolute eosinophils, monocytes, basophils and neutrophils. This predisposes blood recipients to bacterial invasion and increased proliferation of pathogenic bacterial

Table 4 Storage related changes in leucocyte ratios

\begin{tabular}{|c|c|c|c|c|c|c|c|c|}
\hline Leukocyte ratio & Baseline Mean & Week 1 Mean(\% $\Delta)$ & Week 2 Mean $(\% \triangle)$ & Week 3 Mean $(\% \Delta)$ & Week 4 Mean(\% $\triangle)$ & Week 5 Mean $(\% \triangle)$ & $\mathrm{F}$ & $P$-value \\
\hline \multicolumn{9}{|c|}{ Sickle cell and malaria negative donor blood } \\
\hline LER & 19.8 & $20.6(4.0)$ & $20.3(2.5)$ & $21.2(7.1)$ & 22.5(13.6) & $23.9(20.7)$ & 2.36 & 0.052 \\
\hline LMR & 9.7 & 10.0(3.1) & $9.0(7.2)$ & $8.7(10.3)$ & $10.2(5.2)$ & 10.0(3.1) & 0.42 & 0.828 \\
\hline LBR & 184 & 205(11.4) & 128(30.4) & $130(29.3)$ & $150(18.5)$ & 149(19.0) & 0.34 & 0.882 \\
\hline LNR & 0.73 & $0.83(13.7)$ & $1.12(53.4)$ & $1.17(60.3)$ & 1.33(82.2) & $1.88(157.5)$ & 1.44 & 0.222 \\
\hline \multicolumn{9}{|c|}{ Sickle cell trait donor blood } \\
\hline LER & 11.8 & $18.4(55.9)$ & 26.8(127.1) & $36.1(205.9)$ & $38.5(226.3)$ & $32.8(178.0)$ & 5.16 & $0.001^{*}$ \\
\hline LMR & 12.3 & $13.1(6.5)$ & $14.2(15.4)$ & $16.9(37.4)$ & $22.4(82.1)$ & $13.9(13.0)$ & 1.95 & 0.100 \\
\hline LBR & 198 & 177(10.6) & $141(28.8)$ & 169(14.6) & 183(7.6) & 169(14.6) & 0.73 & 0.602 \\
\hline LNR & 0.79 & $1.02(29.1)$ & 1.42(79.8) & $2.22(181.0)$ & $2.83(258.2)$ & $3.23(308.8)$ & 4.53 & 0.001 \\
\hline \multicolumn{9}{|c|}{ Asymptomatic malaria donor blood } \\
\hline LER & 6.8 & $9.2(35.3)$ & 19.3(183.8) & $19.0(179.4)$ & $23.0(238.2)$ & $41.8(514.7)$ & 4.46 & 0.001 \\
\hline LMR & 5.9 & $8.7(47.5)$ & 10.6(79.7) & 11.3(91.5) & 10.6(79.7) & 10.8(83.1) & 2.07 & 0.083 \\
\hline LBR & 79 & $99(25.3)$ & $127(60.8)$ & $175(121.5)$ & 181(129.1) & 199(151.9) & 5.11 & $0.001^{*}$ \\
\hline LNR & 0.32 & $0.56(75.0)$ & $1.07(234.4)$ & $1.42(343.7)$ & $1.27(296.9)$ & $1.72(437.5)$ & 10.9 & $0.001^{*}$ \\
\hline
\end{tabular}

Abbreviations: $L E R$-lymphocytes-to-eosinophils ratio, $L M R$-lymphocytes-to-monocytes ratio, $L B R$-lymphocytes-to-basophils ratio, $L P R$-lymphocyte-to-platelets ratio, $L N R$-lymphocytes-to-neutrophils ratio ${ }^{*} p$-value less than 0.001 
Table 5 Analysis of haemolytic and biochemical parameters in donor blood stored for baseline, week 1, week 3 and week 5

\begin{tabular}{|c|c|c|c|c|c|}
\hline Haemolytic parameters & Baseline Mean & Week 1 Mean $(\% \Delta)$ & Week 3 Mean $(\% \Delta)$ & Week 5 Mean $(\% \triangle)$ & $P$-value \\
\hline \multicolumn{6}{|c|}{ Sickle cell and malaria negative donor blood } \\
\hline Plasma Hb (g/dl) & 0.00 & 0.10 & $0.11(9.1)^{c}$ & $0.21(52.4)^{c}$ & $0.001^{*}, \mathrm{a}$ \\
\hline$\%$ Haemolysis & 0.00 & 0.52 & $0.75(30.7)^{c}$ & $1.44(63.9)^{c}$ & $0.001^{*}, \mathrm{a}$ \\
\hline $\mathrm{LDH}(\mathrm{U} / \mathrm{L})$ & 199.40 & $319.20(60.1)$ & $428.00(114.6)$ & $522.70(162.1)$ & $0.001^{*}, \mathrm{a}$ \\
\hline Potassium (mmol/L) & 4.14 & $5.65(56.5)$ & $7.72(86.5)$ & 10.03(142.3) & $0.001^{*}, \mathrm{~b}$ \\
\hline Sodium (mmol/L) & 137.94 & $134.52(-2.5)$ & $130.00(-5.7)$ & $126.33(-8.4)$ & $0.001^{*}, \mathrm{a}$ \\
\hline Chloride (mmol/L) & 98.66 & $89.06(-3.5)$ & $84.98(-13.9)$ & $83.55(-15.3)$ & $0.001^{*}, \mathrm{~b}$ \\
\hline \multicolumn{6}{|c|}{ Sickle cell trait donor blood } \\
\hline Plasma Hb (g/dl) & 0.00 & 0.24 & $0.37(35.1)^{c}$ & $0.46(47.8)^{c}$ & $0.001^{*}, \mathrm{~b}$ \\
\hline \% Haemolysis & 0.00 & 1.16 & $2.08(44.2)^{c}$ & $2.62(55.7)^{c}$ & $0.001^{*}, \mathrm{a}$ \\
\hline LDH (U/L) & 207.10 & $329.20(59.0)$ & $491.60(137.4)$ & $610.40(194.7)$ & $0.001^{*}, \mathrm{a}$ \\
\hline Potassium (mmol/L) & 4.27 & $6.95(62.8)$ & $12.14(184.3)$ & $20.58(382.0)$ & $0.001^{*}, \mathrm{a}$ \\
\hline Sodium (mmol/L) & 139.51 & $136.74(-2.0)$ & $133.67(-4.2)$ & $130.35(-6.5)$ & $0.001^{*}, \mathrm{a}$ \\
\hline Chloride (mmol/L) & 99.63 & $90.77(-8.9)$ & $83.99(-15.7)$ & $78.50(-21.2)$ & $0.001^{*, b}$ \\
\hline \multicolumn{6}{|c|}{ Asymptomatic malaria donor blood } \\
\hline Plasma Hb (g/dl) & 0.00 & 0.22 & $0.26(15.4)^{c}$ & $0.32(31.3)^{c}$ & $0.001^{*, a}$ \\
\hline$\%$ Haemolysis & 0.00 & 1.00 & $1.46(31.5)^{c}$ & $1.90(47.3)^{c}$ & $0.001^{*}, \mathrm{a}$ \\
\hline $\mathrm{LDH}(\mathrm{U} / \mathrm{L})$ & 192.30 & $418.70(117.7)$ & $596.60(210.4)$ & $806.70(319.5)$ & $0.001^{*}, \mathrm{~b}$ \\
\hline Potassium (mmol/L) & 4.38 & $8.38(91.3)$ & $11.66(166.2)$ & $15.01(242.7)$ & $0.001^{*}, b$ \\
\hline Sodium (mmol/L) & 140.72 & $135.17(-3.9)$ & $130.16(-7.5)$ & $125.60(-10.0)$ & $0.001^{*}$ \\
\hline Chloride (mmol/L) & 100.62 & $91.00(-9.6)$ & $85.71(-14.8)$ & $79.21(-21.3)$ & $0.001^{*}, \mathrm{a}$ \\
\hline
\end{tabular}

Abbreviations: $L D H=$ Lactate dehydrogenase, $S D=$ Standard deviation, $H b=$ Haemoglobin

${ }^{*} p$ values less than $0.001,{ }^{a} p$-value determined by Kruskal-Wallis $\mathrm{H}$ test, ${ }^{b} p$-value determined by one-way ANOVA, ${ }^{c} \% \Delta$ calculated with respect to week 1 mean values

[35, 36]. Fever is a common symptom of sepsis [37]. Donor blood with high LER, LMR, LBR and LNR could cause pathogen induced acute or delayed febrile reactions in recipients with low leukocyte count.

In sickle cell and malaria negative group, red blood cells were relatively stable during storage but in the SCT or asymptomatic malaria donor group, there were significant reduction in red blood cells, haemoglobin and haematocrit. These haematological changes corresponded with gradual increases in plasma haemoglobin due to increased red cell breakdown as well as gradual elevation of potassium ions and lactate dehydrogenase during storage. These observations could be as a result of haemoglobin $S$ erythrocytes fragility subsequent to polymerization of haemoglobin $S$ and reduced deformability and loss of red cell elasticity during storage due to low oxygen tension and low $\mathrm{pH}$ storage medium [38-40] on the one hand, as well as metabolically active intra-erythrocytic Plasmodium parasites could account for these haematological changes. Malaria parasites have been found to be viable in stored blood for at least the first 14 days [41]. When one malaria parasite per microliter of blood is found, that converts to about 500,000 red cells parasitized in a unit of blood [42]. These viable parasites are enough to cause significant haematological derangement in the blood of an infected healthy donor during storage. One of the goals of haemo-transfusion is to restore tissue oxygenation [43]. Stored blood from SCT and asymptomatic malaria donors may develop storage lesions over time due to polymerization of haemoglobin $\mathrm{S}$ in SCT donor blood, loss of deformability and increased osmotic fragility which could compromise their haemorheological properties and oxygen binding and delivery capacity $[44,45]$.

Storage of blood collected from SCT and asymptomatic malaria donors were significantly associated with red cell lysis and elevated plasma haemoglobin. On day of blood collection, donor plasma was free of haemoglobin as well as insignificant differences in potassium, sodium, LDH and chloride in all the donor groups. However at week 1, plasma haemoglobin was 2.4 times higher in SCT donor blood and 2.2 higher in asymptomatic malaria blood compared to sickle cell and malaria negative group. At week 3, plasma haemoglobin was 3.36 times and 2.36 times higher in SCT and asymptomatic malaria donor blood respectively, and at week 5, plasma haemoglobin was 2.2 times higher in SCT and 1.5 higher in asymptomatic malaria group. Excess plasma haemoglobin increased over time in the SCT and 
Table 6 ANOVA analysis of inter-donor category biochemical and haemolytic parameters

\begin{tabular}{|c|c|c|c|c|c|}
\hline Parameters & $\begin{array}{l}\text { Sickle cell/malaria negative } \\
\text { donors Mean } \pm \text { SD }\end{array}$ & $\begin{array}{l}\text { Sickle cell trait donors } \\
\text { Mean } \pm \text { SD }\end{array}$ & $\begin{array}{l}\text { Asymptomatic malaria } \\
\text { donors Mean } \pm \text { SD }\end{array}$ & $\mathrm{F}$ & $P$-value \\
\hline \multicolumn{6}{|l|}{ Baseline } \\
\hline Plasma $\mathrm{Hb}$ & 0.00 & 0.00 & 0.00 & & \\
\hline$\%$ haemolysis & 0.00 & 0.00 & 0.00 & & \\
\hline $\mathrm{LDH}(\mathrm{U} / \mathrm{L})$ & $202.45 \pm 20.51$ & $202.90 \pm 18.91$ & $173.20 \pm 63.74$ & 1.76 & 0.188 \\
\hline Potassium (mmol/L) & $4.15 \pm 0.70$ & $4.22 \pm 0.71$ & $4.01 \pm 1.50$ & 0.39 & 0.681 \\
\hline Sodium (mmol/L) & $138.44 \pm 2.40$ & $139.52 \pm 2.54$ & $126.37 \pm 44.53$ & 3.16 & 0.057 \\
\hline Chloride (mmol/L) & $98.78 \pm 1.66$ & $99.88 \pm 1.78$ & $90.37 \pm 31.87$ & 2.68 & 0.085 \\
\hline \multicolumn{6}{|l|}{ Week 1} \\
\hline Plasma Hb & $0.10 \pm 0.00$ & $0.25 \pm 0.13$ & $0.20 \pm 0.09$ & 23.6 & $0.001^{*}$ \\
\hline$\%$ haemolysis & $0.51 \pm 0.05$ & $1.12 \pm 0.27$ & $1.01 \pm 0.35$ & 20.7 & $0.001^{*}$ \\
\hline LDH (U/L) & $318.36 \pm 29.30$ & $346.00 \pm 78.68$ & $370.90 \pm 137.89$ & 14.6 & $0.001^{*}$ \\
\hline Potassium (mmol/L) & $5.54 \pm 0.41$ & $7.02 \pm 1.15$ & $8.46 \pm 0.75$ & 33.6 & $0.001^{*}$ \\
\hline Sodium (mmol/L) & $134.90 \pm 3.06$ & $136.95 \pm 2.88$ & $121.09 \pm 42.80$ & 1.16 & 0.326 \\
\hline Chloride (mmol/L) & $89.60 \pm 3.12$ & $90.53 \pm 4.19$ & $81.74 \pm 28.74$ & 1.47 & 0.246 \\
\hline \multicolumn{6}{|l|}{ Week 3} \\
\hline Plasma Hb & $0.11 \pm 0.03$ & $0.39 \pm 0.12$ & $0.23 \pm 0.11$ & 22.2 & $0.001^{*}$ \\
\hline$\%$ haemolysis & $0.76 \pm 0.35$ & $2.21 \pm 0.62$ & $1.37 \pm 0.41$ & 17.3 & $0.001^{*}$ \\
\hline $\mathrm{LDH}(\mathrm{U} / \mathrm{L})$ & $436.36 \pm 46.31$ & $491.10 \pm 47.17$ & $605.77 \pm 61.98$ & 33.0 & $0.001^{*}$ \\
\hline Potassium (mmol/L) & $8.05 \pm 1.39$ & $12.02 \pm 1.61$ & $11.83 \pm 1.47$ & 40.3 & $0.001^{*}$ \\
\hline Sodium (mmol/L) & $130.88 \pm 4.23$ & $133.45 \pm 3.42$ & $129.00 \pm 3.51$ & 3.74 & 0.035 \\
\hline Chloride (mmol/L) & $85.61 \pm 3.91$ & $83.37 \pm 3.44$ & $85.37 \pm 2.85$ & 0.73 & 0.487 \\
\hline \multicolumn{6}{|l|}{ Week 5} \\
\hline Plasma Hb & $0.20 \pm 0.04$ & $0.48 \pm 0.19$ & $0.28 \pm 0.14$ & 8.84 & $0.001^{*}$ \\
\hline$\%$ haemolysis & $1.46 \pm 0.51$ & $2.75 \pm 1.00$ & $1.78 \pm 0.65$ & 4.48 & $0.001^{*}$ \\
\hline LDH (U/L) & $536.09 \pm 58.71$ & $631.90 \pm 109.29$ & $798.00 \pm 64.38$ & 13.0 & $0.001^{*}$ \\
\hline Potassium (mmol/L) & $11.35 \pm 4.46$ & $19.48 \pm 2.86$ & $15.18 \pm 1.98$ & 8.25 & $0.001^{*}$ \\
\hline Sodium (mmol/L) & $127.64 \pm 6.00$ & $129.62 \pm 5.17$ & $124.73 \pm 4.79$ & 9.70 & $0.001^{*}$ \\
\hline Chloride (mmol/L) & $84.27 \pm 4.29$ & $77.81 \pm 4.31$ & $78.61 \pm 3.44$ & 1.22 & 0.309 \\
\hline
\end{tabular}

Abbreviations: $L D H=$ Lactate dehydrogenase, $S D=$ Standard deviation, $H b=$ Haemoglobin

${ }^{*} p$-values less than 0.001

the asymptomatic malaria donor groups. At week 1, the percentage haemolysis in the SCT and asymptomatic malaria was more than the permissible level of $0.8 \%$. Blood with \% haemolysis of $0.8 \%$ is not recommended for clinical use [30]. Excess haemoglobin has been found to have negative influence on the intravascular nitric oxide (NO) metabolism after transfusion. Plasma haemoglobin has been found to be a potent scavenger of $\mathrm{NO}$, the most important endogenous vasodilator. In view of this, transfusing blood with high concentration of plasma haemoglobin could decrease NO bioavailability, decreased organ perfusion, increased organ injury [46, 47] and increased mortality in patients with sepsis [48]. Patients with organ failure and patients with septic shock may worsen their condition when transfused with blood with high plasma haemoglobin content. Potassium increased in the SCT and asymptomatic malaria groups and continued till their respective levels were 4.8 and 3.4 times higher than the baseline at week 5 , a factor far more than was observed in the sickle cell/malaria negative group. It is recommended to include malaria and sickle cells screening into donor screening protocols to prevent potassium and free haemoglobin overload. In addition, the clinical impact of transmitting malaria to the recipient, albeit asymptomatic in the donor, merits consideration with recommendations to prevent such transmissions [49].

\section{Conclusion}

Storage of SCT and malaria infected blood affected all the cell lines. At week 1, total white blood cells, neutrophils, red cells, haemoglobin and haematocrit began to fall significantly in SCT and asymptomatic donor blood. 
Significant elevation plasma haemoglobin, increase in \% haemolysis above the permissible level of $0.8 \%$ and potassium elevation above the upper reference range for Ghanaian adults $(5.2 \mathrm{mmol} / \mathrm{L})$ [50] were observed. Significant reduction in red blood cells coupled with significant elevations in plasma haemoglobin, intracellular potassium and lactate dehydrogenase can led to the conclusion that significant number of red cells haemolysis in the experimental set up. This assumption was confirmed by steady elevation of \% haemolysis over the storage weeks.

\section{Limitations of the study}

Malaria parasites were not quantified in the infected blood units. The analysis did not take into consideration the blood group and Rhesus phenotypes of the study units. The gender of the blood donors was unknown. Haemoglobin $\mathrm{S}$ was not quantified in the sickle cell trait donor blood.

\section{Additional file}

Additional file 1: Table S1. Paired sample analysis in haematological parameters among the groups: baseline vs. week 1-3. (DOCX 20 kb)

\begin{abstract}
Abbreviations
2,3 BPG: 2,3-biphosphoglycerate; ANOVA: One-way analysis of variance: ATP: Adenosine triphosphate; Bas: Basophils; CPDA: Citrate Phosphate Dextrose Adenine; Eos: Eosinophils; fL: Fentoliter; GHS: Ghana Health Service; Hb: Haemoglobin; HCT: Haematocrit; LBR: Lymphocytes-to-basophils ratio LER: Lymphocytes-to-eosinophils ratio; LMR: Lymphocytes-to-monocytes ratio; LNR: Lymphocytes-to-neutrophils ratio; Lymp: Lymphocytes; MCH: Mean cell haemoglobin; MCHC: Mean cell haemoglobin concentration; MCV: Mean cell volume; Mon: Monocytes; NBSG: National Blood Service Ghana; Neut: Neutrophils; P_LCR: Platelet large cell ratio; PCT: Plateletcrit; PDW: Platelet distribution width; pg: pictogram; Plt: Platelets; PMV: Mean platelet volume; RBC: Red blood cells; RDW_CV: Red cell distribution width coefficient of variation; RDW_SD: Red cell distribution width standard deviation; SCT: Sickle cell trait; SD: Standard deviation; SSA: sub-Saharan Africa; TTls: Transfusion transmitted infections; TWBC: Total white blood cell
\end{abstract}

\section{Acknowledgements}

The authors are thankful to the management and staff of National blood service, Ghana for collaborating with us in implementing the study. We are also grateful to Linda Baafi (Ga West Municipal Hospital Laboratory) for her voluntary participation in performing the biochemical analysis. Finally we are grateful to the National Malaria Control Programme Ghana for supplying us with malaria rapid diagnostic test kits.

\section{Funding}

The research was 100\% funded by the corresponding author.

\section{Availability of data and materials}

The datasets generated and/or analyzed during the current study are available in Harvard Dataverse repository, doi:https://doi.org/10.7910/DVN/RFBNFN

\section{Authors' contributions}

AE, AP, EYA, AOD conceived, designed the study, collected the data, performed and validated the laboratory findings and drafted the manuscript. TDE performed the statistical analysis. All authors read and approved the final manuscript.

\section{Ethics approval and consent to participate}

Ethical approval of this study was granted by National Blood Service Ghana (NBSGRD/18903/01) and Ghana Health Service Ethical Review Committee (GHSREC002/03/18) approved the study. Consent to participate was not applicable.
Consent for publication

Not applicable.

\section{Competing interests}

The authors declare that they have no competing interests.

\section{Publisher's Note}

Springer Nature remains neutral with regard to jurisdictional claims in published maps and institutional affiliations.

\section{Author details}

'Department of Biomedical Sciences, School of Allied Health Sciences, University of Cape Coast, Cape Coast, Ghana. ${ }^{2}$ School of Public Health, University of Ghana, Legon, Accra, Ghana. ${ }^{3}$ Department of Medical Laboratory Technology, University of Cape Coast, Cape Coast, Ghana.

Received: 19 September 2018 Accepted: 28 October 2018

Published online: 06 November 2018

References

1. Desai M, ter Kuile FO, Nosten F, McGready R, Asamoa K, Brabin B, Newman $\mathrm{RD}$. Epidemiology and burden of malaria in pregnancy. Lancet Infect Dis. 2007;7(2):93-104.

2. Belizario VY, Saul A, Bustos MD, Lansang MA, Pasay CJ, Gatton M, Salazar NP. Field epidemiological studies on malaria in a low endemic area in the Philippines. Acta Trop. 1997:63:241-56.

3. Roucher C, Rogier C, Dieye-Ba F, Sokhna C, Tall A, Trape J-F. Changing malaria epidemiology and diagnostic criteria for Plasmodium falciparum clinical malaria. PLoS One. 2012;7:e46188.

4. da Silva-Nunes M, Ferreira MU. Clinical spectrum of uncomplicated malaria in semi-immune Amazonians: beyond the 'symptomatic' vs 'asymptomatic' dichotomy. Memorias Do Instituto Oswaldo Cruz. 2007;102(3):341-7.

5. Harris I, Sharrock WW, Bain LM, et al. A large proportion of asymptomatic Plasmodium infections with low and sub-microscopic parasite densities in the low transmission setting of Temotu Province, Solomon Islands: challenges for malaria diagnostics in an elimination setting. Malar J. 2010;9:254.

6. Hoyer S, Nguon S, Kim S, Habib N, Khim N, Sum S, et al. Focused screening and treatment (FSAT): a PCR-based strategy to detect malaria parasite carriers and contain drug resistant $P$. falciparum, Pailin, Cambodia. PLoS One. 2012;7(10):e45797.

7. Owusu-Ofori A, Gadzo G, Bates I. Transfusion-transmitted malaria: donor prevalence of parasitaemia and a survey of healthcare workers knowledge and practices in a district hospital in Ghana. Malar J. 2016;15:234-41.

8. Mogtomo ML, Fomekong SL, Kuate HF, Ngane AN. Screening of infectious microorganisms in blood banks in Douala (1995-2004). Sante. 2009;19:3-8.

9. Diop S, Ndiaye M, Seck M, Knight B, Jambou R, Sarr A, Dieye TN, Toure AO, Thiam D, Diakhate L. Prevention of transfusion transmitted malaria in endemic area. Clinical and biological transfusion. 2009:16:454-9.

10. Kinde-Gazard OJ, Gnahoui I, Massougbodji A. The risk of malaria transmission by blood transfusion at Cotonou, Benin. Cahiers Sante. 2000; 10(6):389-92.

11. Oladeinde BH, Omoregie R, Osakue EO, Onaiwu TO. Asymptomatic malaria among blood donors in Benin City Nigeria. Iranian J Parasitol. 2014;9(3):415-22.

12. Aguiar KM, Maia CN. Prevalence of hemoglobin $\mathrm{S}$ in blood donors at the Hemocentro regional in the town of Montes Claros. Minas Gerais RBAC. 2011;43(4):284-7.

13. Antwi-Baffour S, Asare RO, Adjei JK, Kyeremeh R, Adjei DN. Prevalence of hemoglobin $\mathrm{S}$ trait among blood donors: a cross-sectional study. BMC Res Notes. 2015:8:583.

14. Omotade $\mathrm{OO}$, Kayode CM, Falade SL, Ikpeme S, Adeyemo AA, Akinkugbe FM. Routine screening for sickle cell haemoglobinopathy by electrophoresis in an infant welfare clinic. West Afr J Med. 1998;17:91-4.

15. Tubman VN, Marshall R, Jallah W. Newborn screening for sickle cell disease in Liberia: a pilot study. Pediatr Blood Cancer. 2016;63:671-6.

16. Ndeezi G, Kiyaga C, Hernandez AG, Munube D, Howard TA, Ssewanyana I, Nsungwa J, Kiguli S, Ndugwa CM, Ware RE, Aceng JR. Burden of sickle cell trait and disease in the Uganda sickle surveillance study (US3): a crosssectional study. Lancet Global Health. 2016:4:e195-200.

17. Ama V, Kengne AP, Nansseu NJ, Nouthe B, Sobngwi E. Would sickle cell trait influences the metabolic control in sub-Saharan individuals with T2D? Diabet Med. 2012;29:334-7. 
18. Elguero E, Delicat-Loembet $L M$, Rougeron V, Arnathau C, Roche B, Becquart P, Gonzalez JP, Nkoghe D, Sica L, Leroy EM, Durand P, Ayala FJ, Ollomo B, Renaud F, Prugnolle F. Malaria continues to select for sickle cell trait in Central Africa. Proc Natl Acad Sci U S A. 2015;112:7051-4.

19. Grignani C, lamamoto C, Goncalves T, Mashima D, et al. Prevalence of hemoglobin AS among blood donors from Londrina - Parana. RBAC. 2006; 38(4):259-62.

20. Goncalves LB, Duarte EHG, Cabral MD. Prevalence of hemoglobin S in blood donors in the hospital Dr. Agostinho Neto, Praia City - Cape Verde. Sci Public Health. 2015;3(5):600-4.

21. Garba N, Danladi SB, Abubakar HB, Ahmad SG, Gwarzo MY. Distribution of Haemoglobin variants, $A B O$ and $\mathrm{Rh}$ blood groups in blood donors attending Aminu Kano teaching hospital. Clin Med J. 2016:2(2):20-4.

22. Adu P, Simpong DL, Takyi G, Ephraim RKD. Glucose-6-phosphate dehydrogenase deficiency and sickle cell trait among prospective blood donors: a cross-sectional study in Berekum, Ghana. Adv Haematol. 2016; https://doi.org/10.1155/2016/7302912

23. Ghartimagar D. Rational clinical use of blood and blood products - a summary. J Pathol Nepal. 2017:7:1111-7.

24. Adias TC, Moore-lgwe B, Jeremiah ZA. Storage related Haematological and biochemical changes of CPDA-1 whole blood in a resource limited setting. J Blood Disorders Transf. 2012;3:124. https://doi.org/10.4172/2155-9864.1000124.

25. Cheesbrough M. Blood transfusion practice: blood donation and storage of blood, district laboratory practice in tropical countries. Low price edition. Cambridge Universal Press. 2002;2:352-3.

26. Koch CG, Figueroa PI, Li L, et al. Red blood cell storage: how long is too long? Ann Thorac Surg. 2013;96(5):1894-9.

27. Bonaventura J. Clinical implications of the loss of vasoactive nitric oxide during red blood cell storage. Proc Natl Acad Sci U S A. 2007;104:19165-6.

28. Spinella PC, Sparrow RL, Hess JR, et al. Properties of stored red blood cells: understanding immune and vascular reactivity. Transfusion. 2011;51(4):894-900.

29. WHO. Guidelines on assessing donor suitability for blood donation. World Health Organization 2012. http://www.who.int/iris/handle/10665/76724. Assessed 7 Aug 2018.

30. Sawant RB, Jathar SK, Rajadhyaksha SB, Kadam PT. Red cell hemolysis during processing and storage. Asian J Transfus Sci. 2007:1(2):47-51.

31. Koch CG, Li L, Sessler DI, Figueroa P, Hoeltge GA, et al. Duration of red-cell storage and complications after cardiac surgery. N Engl J Med. 2008;358: 1229-39.

32. van de Watering L. Red cell storage and prognosis. Vox Sang. 2011;100:36-45.

33. Hess JR. Red cell changes during storage. Transfus Apher Sci. 2010;43:51-9.

34. Hess JR. An update on solutions for red cell storage. Vox Sang. 2006;91:13-9.

35. Antoniadou A, Giamarellou H. Fever of unknown origin in febrile leukopenia. Infect Dis Clin N Am. 2007;21(4):1055-90.

36. Friese CR. Chemotherapy-induced neutropenia: important new data to guide nursing assessment and management. Cancer Ther Support Care 2006:4(2):21-5.

37. Schortgen F. Fever in sepsis. Minerva Anestesiol. 2012;78(11):1254-64.

38. Brandao MM, Saad O, Cezar CL, Fontes A, Costa FF, Barjas-Castro ML. Elastic properties of stored red blood cells from sickle trait donor units. Int J Transfusion Med. 2003. https://doi.org/10.1046/j.1423-0410.2003.00344.x.

39. Noguchi CT, Torchia DA, Schechter AN. Polymerization of hemoglobin in sickle cell trait erythrocytes and lysate. J Biol Chem. 1981;256:4168-71.

40. Helzlsouer KJ, Hayden FG, Rogol AD. Severe metabolic complications in a cross country runner with sickle cell trait. JAMA. 1983;249:777-9.

41. Kitchen AD, Chiodini PL. Malaria and blood transfusion. Vox Sang. 2006; 90(2):77-84.

42. Owusu-Ofori AK, Betson M, Parry CM, Stothard R, Bates I. Transfusiontransmitted malaria in Ghana. Clin Infect Dis. 2013:56(12):1735-41.

43. Raat NJ, Ince C. Oxygenating the microcirculation: the perspective from blood transfusion and blood storage. Vox Sang. 2007;93:12-8.

44. Kim-Shapiro DB, Lee J, Gladwin GT. Storage lesions: role of red blood cell breakdown. Transfusion. 2011;51:844-51.

45. Aubron C, Nichol A, Cooper DJ, Bellomo R. Age of red blood cells and transfusion in critically ill patients. Ann Intensive Care. 2013. https://doi.org/ 10.1186/2110-5820-3-2.

46. Donadee C, Raat NJ, Kanias T, Tejero J, Lee JS, Kelley EE, Zhao X, Liu C, Reynolds H, Azarov I, Frizzell S, Meyer EM, Donnenberg AD, Qu L, Triulzi D, Kim-Shapiro DB, Gladwin MT. Nitric oxide scavenging by red blood cell microparticles and cell-free hemoglobin as a mechanism for the red cell storage lesion. Circulation. 2011;26:465-76.
47. Minneci PC, Deans KJ, Zhi H, Yuen PS, Star RA, Banks SM, Schechter AN, Natanson C, Gladwin MT, Solomon SB. Hemolysis-associated endothelia dysfunction mediated by accelerated $\mathrm{NO}$ inactivation by decompartmentalized oxyhemoglobin. J Clin Invest. 2005;115:3409-17.

48. Adamzic M, Hamburger T, Petrat F, Peters J, de Groot H, Hartmann M. Free hemoglobin concentration in severe sepsis: methods of measurement and prediction of outcome. Crit Care. 2012. https://doi.org/10.1186/cc11425.

49. Infectious disease testing for blood transfusions. NIH Consensus Panel on Infectious Disease Testing for blood transfusions. JAMA. 1995;274(17):1374-9.

50. Dosoo DK, Adu-Gyasi D, Kwara E, Ocran J, Osei-Kwakye, et al. Haematological and biochemical reference values for healthy adults in the middle belt of Ghana. PLOS ONE. 2012;7(4):e36308. https://doi.org/10.1371/ journal.pone.0036308.
Ready to submit your research? Choose BMC and benefit from:

- fast, convenient online submission

- thorough peer review by experienced researchers in your field

- rapid publication on acceptance

- support for research data, including large and complex data types

- gold Open Access which fosters wider collaboration and increased citations

- maximum visibility for your research: over $100 \mathrm{M}$ website views per year

At BMC, research is always in progress.

Learn more biomedcentral.com/submissions 